\title{
Front Matter: Volume 10453
}

, "Front Matter: Volume 10453," Proc. SPIE 10453, Third International Conference on Applications of Optics and Photonics, 1045301 (22 August 2017); doi: 10.1117/12.2287443

SPIE Event: Third International Conference on Applications of Optics and Photonics, 2017, Faro, Portugal 


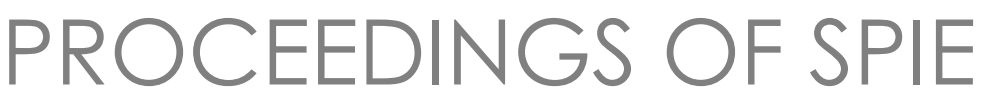

\title{
Third International Conference on Applications of Optics and Photonics
}

\author{
Manuel F. M. Costa \\ Editor
}

\author{
8-12 May 2017 \\ Faro, Portugal \\ Organized by \\ SPOF-Sociedade Portuguesa para a Investigação e Desenvolvimento em Óptica e Fotónica \\ "Portuguese Society for Optics and Photonics" (Portugal) \\ Sponsored by \\ ICO-International Commission for Optics • EOS-European Optical Society • \\ RIAO/SOPHIA-Red(e) Iberoamericana de Óptica • SEDOPTICA-Sociedad Española de \\ Óptica (Spain) • AMO-Academia Mexicana de Óptica (Mexico) • RCO-Red Colombiana \\ de Óptica (Colombia) - OPSS-Optics and Photonics Society of Singapore (Singapore) \\ CVO-Comité Venezolano de Optica (Venezuela) • Innova Scientific, S.L. (Spain) • M.T. \\ Brandão, Lda. (Portugal) • SOFE - Sociedad de Optica Y Fotonica del Ecuador (Ecuador)
}

Cooperating Organization and Publisher

SPIE

Volume 10453 
The papers in this volume were part of the technical conference cited on the cover and title page. Papers were selected and subject to review by the editors and conference program committee. Some conference presentations may not be available for publication. Additional papers and presentation recordings may be available online in the SPIE Digital Library at SPIEDigitallibrary.org.

The papers reflect the work and thoughts of the authors and are published herein as submitted. The publisher is not responsible for the validity of the information or for any outcomes resulting from reliance thereon.

Please use the following format to cite material from these proceedings:

Author(s), "Title of Paper," in Third International Conference on Applications of Optics and Photonics, edited by Manuel F. M. Costa, Proceedings of SPIE Vol. 10453 (SPIE, Bellingham, WA, 2017) Seven-digit Article CID Number.

ISSN: 0277-786X

ISSN: 1996-756X (electronic)

ISBN: 9781510613836

ISBN: 9781510613843 (electronic)

Published by

SPIE

P.O. Box 10, Bellingham, Washington 98227-0010 USA

Telephone +1 3606763290 (Pacific Time) · Fax +1 3606471445

SPIE.org

Copyright (@ 2017, Society of Photo-Optical Instrumentation Engineers.

Copying of material in this book for internal or personal use, or for the internal or personal use of specific clients, beyond the fair use provisions granted by the U.S. Copyright Law is authorized by SPIE subject to payment of copying fees. The Transactional Reporting Service base fee for this volume is $\$ 18.00$ per article (or portion thereof), which should be paid directly to the Copyright Clearance Center (CCC), 222 Rosewood Drive, Danvers, MA 01923. Payment may also be made electronically through CCC Online at copyright.com. Other copying for republication, resale, advertising or promotion, or any form of systematic or multiple reproduction of any material in this book is prohibited except with permission in writing from the publisher. The CCC fee code is $0277-786 \mathrm{X} / 17 / \$ 18.00$.

Printed in the United States of America.

Publication of record for individual papers is online in the SPIE Digital Library.

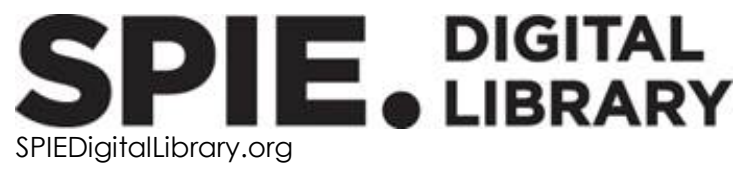

Paper Numbering: Proceedings of SPIE follow an e-First publication model. A unique citation identifier (CID) number is assigned to each article at the time of publication. Utilization of CIDs allows articles to be fully citable as soon as they are published online, and connects the same identifier to all online and print versions of the publication. SPIE uses a seven-digit CID article numbering system structured as follows:

- The first five digits correspond to the SPIE volume number.

- The last two digits indicate publication order within the volume using a Base 36 numbering system employing both numerals and letters. These two-number sets start with 00, 01, 02, 03, 04, 05, 06, 07, 08, 09, OA, OB ... 0Z, followed by 10-1Z, 20-2Z, etc. The CID Number appears on each page of the manuscript. 


\title{
Contents
}

\author{
xi Authors \\ xv Conference Committees \\ xx Introduction
}

THIRD INTERNATIONAL CONFERENCE ON APPLICATIONS OF OPTICS AND PHOTONICS

1045302 Interchannel collisions of strong dispersion-managed solitons with different energies in the presence of higher-order effects [10453-2]

1045303 Optical microtopographic inspection of asphalt pavement surfaces [10453-3]

1045304 Spectrophotometric characterization of hemozoin as a malaria biomarker [10453-5]

1045305 Low cost ellipsometer using a standard commercial polarimeter [10453-6]

1045306 Generation of five phase-locked harmonics in the continuous wave regime and its potential application to arbitrary optical waveform synthesis [10453-7]

1045307 Resonant tunneling diode photodetectors for optical communications [10453-9]

1045308 Modelling of optoelectronic circuits based on resonant tunneling diodes [10453-10]

1045309 Optical bias selector based on a multilayer a-SiC:H optical filter [10453-11]

10453 OA Visible light communication and indoor positioning using a-SiCH device as receiver [10453-12]

10453 OB Low-cost fused taper polymer optical fiber (LFT-POF) splitters for environmental and home-networking solution [10453-13]

10453 OC Goos-Hänchen shift of Cosine-Gaussian Schell-model beams with rectangular symmetry [10453-19]

10453 OD Simulation of localized surface plasmon in metallic nanoparticles embedded in amorphous silicon [10453-21]

10453 OE Mode-locked semiconductor laser for long and absolute distance measurement based on laser pulse repetition frequency sweeping: a comparative study between three types of lasers [10453-22]

10453 OF Erbium and ytterbium co-doped transparent oxyfluoride glass-ceramics optical fibers [10453-24] 
10453 OG Fabrication and characterization of a cell electrostimulator device combining physical vapor deposition and laser ablation (Best Student Paper) [10453-25]

$10453 \mathrm{OH} \quad$ Indoors positioning through VLC technology using an a-SiC:H photodetector [10453-26]

$10453 \mathrm{Ol}$ Critical discussion on the UV absorption properties of Earth's atmosphere [10453-28]

10453 0J Energy transfer between silver clusters and europium Eu ${ }^{3+}$ ions in photo-thermo-refractive glasses [10453-30]

10453 OK Optical, colloidal and biological properties of up-converting nanoparticles embedded in polyester nanocarriers [10453-32]

$10453 \mathrm{OL}$ Indirect gonioscopy system for imaging iridocorneal angle of eye [10453-35]

10453 OM On the behavior of linear polarizers on highly focused radially polarized beams [10453-39]

10453 ON Laser based manufacturing of channels and improvement of their lifetime with sol-gel coatings [10453-40]

1045300 Validation of a semi-automatic protocol for the assessment of the tear meniscus central area based on open-source software [10453-41]

10453 OP Ferrofluids with high dynamic ranges of optical transmission [10453-43]

$104530 Q \quad$ Photonic integrated circuits for NG-EPON [10453-48]

10453 OR Special microstructured fibers with irregular and regular claddings for supercontinuum generation [10453-49]

10453 OS Modeling multicore integrated waveguides in highly doped glass [10453-50]

10453 OT Clinical performance of an objective methodology to categorize tear film lipid layer patterns [10453-51]

10453 OU Color-quality control using color-difference formulas: progress and problems (Invited Paper) [10453-56]

10453 OV Electromagnetic properties of a monolayer of polarisable particles deposited on graphene (Runner Up Best Student Paper) [10453-59]

10453 OW Quantification of the absorption of light in coals using fiber optic sensors [10453-60]

10453 OX Modeling of dynamics of nanosecond laser ablation in phase explosion regime [10453-61]

10453 OY Continual and molecular dynamics approaches in determining thermal properties of silicon [10453-62]

$104530 Z$ Electromagnetic simulation of amorphous silicon waveguides [10453-63]

1045310 Wavemeter improvements for laser diode calibration (Invited Paper) [10453-65] 
1045311 Space-time refraction of light in time dependent media: the analogue within the analogue (Invited Paper) [10453-66]

1045312 Surface enhanced Raman spectroscopy analysis of HeLa cells using a multilayer substrate [10453-67]

1045313 Scattering, absorption and transmittance of experimental graphene dental nanocomposites [10453-68]

1045314 The analogue quantum mechanical of plasmonic atoms [10453-74]

1045315 Solving the multi-level Maxwell-Bloch equations using GPGPU computing for the simulation of nonlinear optics in atomic gases [10453-77]

1045316 Doppler broadening effects in plasmonic quantum dots [10453-78]

1045317 Development of a quantum particle in cell algorithm in GPU for solving Maxwell-Bloch equations [10453-79]

1045318 Fast physical ray-tracing method for gravitational lensing using heterogeneous supercomputing in GPGPU [10453-80]

1045319 Ultrasensitive detection of phenolic antioxidants by surface enhanced Raman spectroscopy [10453-81]

104531 A Tunable light fluids using quantum atomic optical systems [10453-84]

10453 1B Pinching optical potentials for spatial nonlinearity management in Bose-Einstein condensates [10453-85]

10453 1C Dissipative solitons in 4-level atomic optical systems [10453-86]

10453 ID Wavefront coding for visual optics [10453-87]

10453 IE SPaCe-GEM: solver of the Einstein equations using GPUs under the gravitoelectromagnetic approximation [10453-90]

10453 IF The extended Kubelka-Munk theory and its application to colloidal systems [10453-92]

$104531 G$ Modelling metal-dielectric core-shell nanoparticles with effective medium theories [10453-93]

$10453 \mathrm{1H}$ Image encryption scheme based on computer generated holography and time-averaged moiré [10453-94]

1045311 Effect of cooling water on ablation in Er:YAG laserosteotome of hard bone [10453-95]

$104531 \mathrm{~J}$ Mueller matrix imaging and analysis of cancerous cells [10453-97]

$104531 \mathrm{~K}$ Evaluation of FEL lamp seasoning behavior for use as a spectral irradiance standard [10453-98] 
$104531 \mathrm{~L} \quad$ Physical ray-tracing method for anisotropic optical media in GPGPU [10453-99]

$104531 \mathrm{M}$ First approach on the Haar Transform applied with a 2 x 2 multimode interferometer [10453-100]

10453 iN Partially polarized pseudo-Schell model sources [10453-102]

1045310 Studying tunability of some NIR semiconductor lasers by external cavity setup [10453-103]

$104531 \mathrm{P} \quad$ Interocular suppression [10453-108]

$104531 Q \quad$ Optical quasi-distributed simultaneous vibration and temperature sensing in stator bars of a 370-MVA electric generator [10453-110]

10453 1R Refractive index sensitivity in etched FBG in the visible range [10453-114]

10453 is Effect of laser pulse duration on ablation efficiency of hard bone in microseconds regime [10453-115]

$104531 \mathrm{1T}$ Towards the automatization of the Foucault knife-edge quantitative test [10453-1 18]

$104531 \mathrm{U}$ Simulation of partially coherent light propagation using parallel computing devices [10453-120]

10453 IV Study of photo activation reaction of experimental graphene dental nanocomposites through dynamic laser speckle [10453-123]

10453 IW Variable structure and sliding modes nonlinear control system applied to a fiber optic interferometer [10453-124]

$104531 \mathrm{~A}$ A model for prediction of color change after tooth bleaching based on CIELAB color space [10453-125]

$104531 \mathrm{Y} \quad$ Finite element model for the simulation of laser activated micro- and nano-scale drug delivery systems [10453-126]

1045312 Short-length long period fiber grating for torsion sensing applications [10453-127]

1045320 Spatio-temporal processing of ultra-short pulses with micro optics (Invited Paper) [10453-129]

$1045321 \quad$ Fabry-Perot interferometer based on array of microspheres for temperature sensing (Invited Paper) [10453-136]

1045322 Using DSLR cameras in digital holography [10453-137]

1045323 Full Poincaré beams obtained by means of uniaxial crystals [10453-140]

1045324 Simultaneous measurement of temperature and refractive index based on microfiber knot resonator integrated in an abrupt taper Mach-Zehnder interferometer [10453-141]

1045325 Thermal sensitivity increase of RFBG in the visible range [10453-142] 
1045326 Strain sensor based on hollow microsphere Fabry-Perot cavity [10453-144]

1045327 Dental resins properties studied by Bragg gratings (Invited Paper) [10453-146]

1045328 Infrared light sensor applied to early detection of tooth decay [10453-148]

1045329 Influence of a perturbation in the Gyrator domain for a joint transform correlator-based encryption system [10453-153]

$104532 \mathrm{~A}$ The USC-OSA-EPS section activities in optics [10453-154]

10453 2B Direct formation of nanostructures by focused electron beam on a surface of thin metallic films [10453-156]

10453 2C Rapid Eye Movements (REMs) and visual dream recall in both congenitally blind and sighted subjects (Invited Paper) [10453-158]

$104532 D \quad$ Assessing the wavefront aberrations of the emmetropic eye after a reading task [10453-161]

$104532 \mathrm{E} \quad$ Strong coupling effects in hybrid plexitonic systems (Invited Paper) [10453-163]

10453 2G An exploratory study of temporal integration in the peripheral retina of myopes [10453-171]

$104532 \mathrm{H} \quad$ Organic semiconductor rubrene thin films deposited by pulsed laser evaporation of solidified solutions [10453-176]

104532 MIMO processing based on higher-order Poincaré spheres [10453-177]

$104532 \mathrm{~J} \quad$ Optical properties of an anterior lamellar human cornea model based on fibrin-agarose [10453-179]

$104532 \mathrm{~K} \quad 2.05-\mu \mathrm{m}$ Holmium-doped all-fiber continuous-wave laser at in-core diode-pumping at $1.125 \mu \mathrm{m}[10453-180]$

$104532 \mathrm{~L} \quad$ Evaluating polarization diversity for speckle reduction in retinal image quality estimates [10453-183]

$104532 \mathrm{M} \quad$ Researching in biomaterials optics (Invited Paper) [10453-186]

$104532 \mathrm{~N}$ Modeling and possible implementation of self-learning equivalence-convolutional neural structures for auto-encoding-decoding and clusterization of images [10453-187]

1045320 Photo-induced ultrasound microscopy for photo-acoustic imaging of non-absorbing specimens [10453-189]

$104532 \mathrm{P} \quad$ Electrical addressing and temporal tweezing of localized pulses in passively mode-locked semiconductor lasers (Invited Paper) [10453-190]

$104532 \mathrm{R}$ Resonant tunneling diode oscillators for optical communications [10453-193] 
$104532 R$ White light spectral interferometer for measuring dispersion in the visible-near infrared [10453-199]

1045325 Impact of different environmental conditions on lithium-ion batteries performance through the thermal monitoring with fiber sensors [10453-200]

$104532 \mathrm{~T}$ Phase contrast imaging of red blood cells using digital holographic interferometric microscope [10453-202]

$104532 \mathrm{U}$ Fourier transform phase difference method optimization for supersonic gas flow characterization [10453-203]

$104532 \mathrm{~V}$ Fabry-Perot cavity based on air bubble for high sensitivity lateral load and strain measurements [10453-204]

$104532 \mathrm{~W}$ Polymer and tapered silica fiber connection for polymer fiber sensor application [10453-205]

$104532 X$ Onion cell imaging by using Talbot/self-imaging effect [10453-208]

$104532 Y$ Resonant tunnelling diode based high speed optoelectronic transmitters [10453-209]

$104532 Z$ THz metrology for active electronic devices: state of the art and challenges [10453-211]

1045330 Effect of periodicity in the optimization of fine tuned dipolar plasmonic structures for SERS [10453-213]

1045331 An advanced arrangement of the optical spectrograph based on acousto-optical and cross-disperser techniques for astronomical applications [10453-217]

1045332 Analytical transient analysis of Peltier device for laser thermal tuning [10453-221]

1045333 Wavelength tuning of polymer optical fibre Bragg grating at longer wavelengths permanently [10453-224]

1045334 Novel multiband polarization beam splitter based on a dual-core transversally chirped microstructured optical fiber [10453-229]

1045335 Electro-thermal effects in large area white-organic light emitting diodes [10453-230]

1045336 Nonlinear joint transform correlator architectures for images encryption, decryption and authentication systems [10453-236]

1045337 Design of low loss photonic crystal fiber based on porous-core with elliptical holes in THz regime [10453-237]

1045338 Detection of low frequency, out-of-plane vibrations by the Talbot effect and adaptive photodetectors [10453-238] 
1045339 Multi-arm spectrometer for parallel frequency analysis of radio-wave signals oriented to astronomical observations [10453-240]

10453 3A Usage of CISS and Conlon surveys in eye accommodation studies [10453-241]

Proc. of SPIE Vol. 10453 1045301-9

Downloaded From: https://www.spiedigitallibrary.org/conference-proceedings-of-spie on 26 Apr 2023 Terms of Use: https://www.spiedigitallibrary.org/terms-of-use 


\section{Authors}

Numbers in the index correspond to the last two digits of the seven-digit citation identifier (CID) article numbering system used in Proceedings of SPIE. The first five digits reflect the volume number. Base 36 numbering is employed for the last two digits and indicates the order of articles within the volume. Numbers start with 00, 01, 02, 03, 04, 05, 06, 07, 08, 09, 0A, 0B...0Z, followed by 10-1Z, 20-2Z, etc.

Abbasi, Hamed, 11, 10, 1s

Ab-Rahman, Mohammad Syuhaimi, OB

Abreu, Manuel, 05, OE

Acosta, E., 1D

Afseth, N., 19

Agarwal, Shilpi, $2 X$

Aguilar-Hernández, I. A., 12, 19

Aizpurua, Javier, 2E

Alaminos, Miguel, 2J

Alcaraz de la Osa, R., 1F, $1 G$

Aldana, Mikel, 2L

Alegria, E., OD

Algnamat, Bilal S., $2 R$

Alharbi, Khalid H., 07

Al-Khalidi, Abdullah, 07, 2Q, 2Y

Almaguer, C., 1D

Almeida, A. L., 1C

Almeida, Álvaro J., 32

Álvarez, Ezequiel, ON

Alves, R. A., 14, 15, 16, 17, 18, 1A, 1B, 1C, 1E, 1L

Amorín, Adán, 2A

Antona, Beatriz, 2C

Aragón, Ángel L., OG, 2A

Arellanes, Adan Omar, 31, 39

Arines, J., 1D

Arosa, Yago, 2R

Augustyn, Elżbieta, OF

Aung, Tin, OL

Aymerich, María, ON, 2A

Bani, Mohammad Amin, 10

Bao-Varela, Carmen, 0G, 2A

Baptista, António M. G., 2G

Barmenkov, Yuri O., 2K

Barrio, Ana, 2C

Baskaran, Mani, OL

Bastos, Ricardo, 32

Bazylińska, Urszula, OK

Beltrán, Lina, 1I, 1S

Benedicto, David, OS

Benjumea, Eberto, 28

Berbel, M. A., OC

Bértolo, Helder, 2C

Bierlich, Jörg, 21

Blanco, Manuel, 2A

Blanco García, Jesús, 10, $2 U$

Botero-Cadavid, Juan F., 37

Cabral, Alexandre, $\mathrm{OE}$

Camelin, P., 2P

Cambronero-López, Ferran, 2A
Cantu, Horacio, $2 Q$

Cardona, Juan de la Cruz, 2J, 2M

Cardozo da Silva, Jean Carlos, $1 Q$

Carnicer, Artur, OM

Castañeda-Quintero, Raul, 2L

Castro Alves, D., OE

Catarino, Susana O., 04

Cattin, Philippe C., 11, 1S

Cerezo, V., 03

Chavez Dagostino, Miguel, 39

Chavushyan, Vahram, 31

Chiamenti, Ismael, 1R, 25

Coelho, João M. P., 1Y, 1Z

Contreras-Torres, F. F., 19

Correa, Nelson, 2L

Costa, J. C., OH, 11, 14, 15, 16, 17, 18, 1A, 1B, 1C, $1 \mathrm{E}, 1 \mathrm{~L}$

Costa, Manuel F. M., 03

Cui, G., OU

Cunillera, A., OC

Czerska, Elwira, OF

de la Fuente, Raul, 2R

de la Rosa, I., IT

De La Rue, Richard M., 30

Demin, M. M., OX

Delgado, Tamara, 2A

de Moura, Camila Carvalho, 25

de Oliveira, Valmir, 25

de Sande, Juan Carlos G., 1N, 23

Díaz, Leonardo, 0W, 28

Diaz-Otero, F. J., 02

Diz-Bugarin, J., 10

Döhring, Thorsten, 0 l

Dreyer, Uilian José, $1 Q$

Dudea, Diana, 13

Encarnação, Tito J., $2 \mathrm{G}$

Espitia, José, 28

Esteban, Ruben, 2E

Fantoni, Alessandro, OA, OD, OZ

Feldmann, Jochen, 2E

Fernandes, Gil M., 2l

Fernandes, João, 1M

Fernandes, M., OD

Fernández, A., 1F, $1 \mathrm{~J}$

Fernández-Luna, J. L., $1 \mathrm{~J}$

Fernández-Maloigne, C., OU

Ferreira, Antonio F. G., Jr., $1 \mathrm{~K}$

Ferreira, Marta S., 21, 2S, 2V

Ferreira, Miguel F. S., 2W 
Ferreira, T. D., 1A

Figueiredo, José M. L., 07, 08, 2Q, 2Y

Flores-Arias, M. Teresa, ON, 2A

Foot, James A., 07, 08

Franco, Sandra, 2D

Frazão, Orlando, 21, 24, 26, 2W

Freitas, E. F., 03

Friederich, N., is

Fu, Chan Yiu, OL

Gallego, Daniel, 33

García, P. A., OU

Garcia-Resua, Carlos, 00, 0T

García-Sucerquia, Jorge, 22

Gargello, Ana, 2A

Garzón, Ingrid, 2J

Gavara, T., 06

Gaviria, Jefry, 2L

Gazda, M., 2H

Gebert de Oliveira Franco, Ana Paula, 27

Gerbreders, Vjaceslavs, 2B

Ghafoor, Moeen, 0J

Ghinea, Razvan, 13, 2J, 2M

Giraldez, Maria J., 00, OT

Giudici, M., 2P

Gomes, André D., 24, 2W

Gomes, J., 35

Gomes, M., 11, 14, 15, 16, 17, 18, 1A, 1B, 1C, 1E,

$1 \mathrm{~L}$

Gómez-Varela, Ana I., ON, 2A

Gomez, Freiman, 34

Gómez-Cardona, N., 34

Gómez-Robledo, L., OU

González, F., 1F, 1 G

González-Andrades, Miguel, 2J

Gori, Franco, $1 \mathrm{~N}$

Govyadinov, Alexander A., 2E

Grzelczak, Marek, 2E

Guerreiro, A., 11, 14, 15, 16, 17, 18, 1A, 1B, 1C, 1E, $1 \mathrm{~L}$

Guillan-Lorenzo, O., 02

Gunab, Hadi, OB

Gutiérrez, Y., 1F, 1G

Guzman, Raphael, 11

Hänninen, Pekka E., 20

Harun, Mohd Hazwan, OB

Herrera, Luis J., 1X, 2M

Herrera-Ramírez, Jorge, 22, 2L

Hincapié-Zuluaga, Diego, 22, 2L

Holmes-Smith, A. Sheila, 30

Hong, F.-L., 06

Huang, M., OU

Illarramendi, Maria Asunción, OR

Ionescu, Ana M., 2J, 2M

Ivanov, R., $1 T$

Jahns, J., 20

Janusas, Giedrius, $1 \mathrm{H}$

Jaschinski, Wolfgang, 3A

Javaloyes, J., 2P

Jendrzejewski, R., $2 \mathrm{H}$

Johnson, Nigel P., 30
Juvells, Ignasi, OM

Kalinowski, Hypolito José, 1R, 25, 27

Kamicawachi, Ricardo C., IR

Karam, Leandro Zen, 27

Katsuragawa, M., 06

Kelly, Anthony E., 2Q

Kępińska, Mirosława, OF

Khan, Gufran S., 2T

Kir'yanov, Alexander V., 2K

Kitano, Cláudio, $1 \mathrm{~W}$

Kobelke, Jens, 21

Koepke, Czesław, OF

Koho, Sami $\vee ., 20$

Korneev, N., 38

Koroleva, O. N., OY

Kowal, Dominik, 2W

Krasilenko, Vladimir, G., 2N

Krumina, Gunta, 3A

Kuhne, Jean F., 1R

Kulbacka, Julita, OK

Kumar, M., 35

Kumar, Varun, 2T, $2 \mathrm{X}$

Lamela Rivera, Horacio, 33

Lazarev, Alexander, A., 2N

Li, C., OU

Lima, Mário, OQ, 1M

Lima, Rui, 04

Lisiecki, Radosław, OF

Liz-Marzán, Luis M., 2E

López-Lago, Elena, 2R

López-Luke, T., 12, 19

Loren Inácio, Patrícia, 1R, 25

Lourenço, Paulo, $\mathrm{OZ}$

Louro, P., 09, OA, OD, OH

Lucena, Cristina, IV

Macedo, Antonio F., $2 \mathrm{G}$

Magalhães, Tiago C., $1 U$

Majewska, N., $2 \mathrm{H}$

Majumdar, $\mathrm{S} ., 2 \mathrm{H}$

Maluenda, David, OM, $1 \mathrm{~N}$

Marasanov, Dmitriy, OJ

Marconi, M., 2P

Martelli, Cicero, $1 Q$

Martín, J. C., OP

Martin, Roberta I., IW

Martínez, G., $1 T$

Martínez-Herrero, Rosario, OC, OM, IN

Mazhukin, A. V., OX, OY

Mazhukin, V.I., OX, OY

McMeekin, Scott G., 30

Melgosa, M., OU

Melnikau, Dzmitry, 2E

Mendonça, J. T., 11

Mergo, Pawel, $2 \mathrm{~W}$

Mestre, Tiago, 2C

Millán, María S., 29, 36

Minas, Graça, 04

Minkovich, Vladimir P., OR

Moldovan, Marionara, 13

Monteiro, Catarina S., 26 
Moraes, José Carlos T. B., 1K

Moreno, F., IF, 1G, 1J

Morillas, S., OU

Muga, Nelson J., 21

Murukeshan, V. M., OL

Nadas, Rafael B., $1 R$

Naftaly, Mira, $2 Z$

Naim, Nani Fadzlina, OB

Nakagawa, K., 06

Nascimento, Micael, 25

Nazeri, Majid, 10

Nespereira, Marta, $1 Z$

Nieto, Daniel, OG

Nikitovich, Diana V., 2N

Nikonorov, Nikolay, 0J

Novais, Susana, 2V

Nunes, Amélia Fernandes, IP

Ohae, C., 06

Oliveira, Cristina M., 2D

Ornelas-Soto, N., 12, 19

Ortiz, D., IF, IG

Outumuro, I., 10

Palevicius, Arvydas, $1 \mathrm{H}$

Palevicius, Paulius, $1 \mathrm{H}$

Panke, Karola, 3A

Pazos, Antonio, OG

Pecho, Óscar E., 2M

Pedrosa-Rodriguez, L., 02

Pena-Verdeal, Hugo, 00, ОT

Pereira, L., 35

Pereira, Rui M. S., OV

Peres, Nuno, OV

Pérez, Eliseo, OG

Pérez, María del Mar, 13, 1V, 1X, 2J, 2M

Pérez-Cabré, Elisabet, 29, 36

Perinchery, Sandeep M., OL

Pichardo-Molina, J. L., 12

Pinho, Pedro, $\mathrm{OZ}$

Pinto, A., 35

Pinto, Armando N., 2

Pinto, João L., 2S, 2V

Piquero, Gemma, 1N, 23

Pospori, Andreas, 33

Pozo, Antonio M., $1 \mathrm{~V}$

Pulgar, Rosa, $1 \mathrm{X}$

Ragulskis, Minvydas, $1 \mathrm{H}$

Rakovich, Yury P., 2E

Rauter, Georg, 11, 15

Rebordão, José Manuel, OE, IU, 1Y, 12

Rei, João F. M., 07, 08

Reyes-Vera, Erick, 34, 37

Ribeiro, A., OD

Richard, N., OU

Rivas, María José, 1X, 2M

Rodrigues, Carla, $\mathrm{OQ}$

Rodrigues, Francisco, OQ, $1 M$

Rodrigues, Gil C., 07, 08, 2 Y

Rodríguez Fernández, Carlos Damian, 2R

Rodríguez Lorenzo, Francisco, $2 \mathrm{U}$

Rodríguez, G., $1 T$
Rodríguez-Montero, P., 38

Sagias, Georgios, 33

Saiz, J. M., 1F, 1G, 1J

Sakamoto, João M. S., IW

Salas, Marianne, 13, 1V

Sánchez-Iglesias, Ana, 2E

Sánchez-García, Ángel, 2A

Santana, Janiley, $1 \mathrm{X}$

Santarsiero, Massimo, 1N, 23

Santos, Jaime E., OV

Santos, José L., 21

Sanz, J. M., $1 G$

Sanz-Felipe, Á., OP

Savateeva, Diana, 2E

Sawczak, M., 2H

Schmidt, Mikolaj K., 2E

Sgibnev, Yevgeniy, OJ

Shahpari, Ali, 32

Shakher, Chandra, 2T, 2X

Shapranov, A. V., OX, OY

Sharp, Graham G., 30

Shcherbakov, Alexandre S., 31, 39

Sheikhnejad, Yahya, 32

Silva, Ivo, 04

Silva, Nuno A., 11, 14, 15, 16, 17, 18, 1A, 1B, 1C, $1 \mathrm{E}, 1 \mathrm{~L}$

Silva, Susana O., 26

Simon, Thomas, 2E

Śliwiński, G., $2 \mathrm{H}$

Snikeris, Janis, 2B

Sotsky, Alexander B., OR

Souto, André, OV

Statkiewicz-Barabach, Gabriela, 2W

Suhaimi, N. Sheeda, 06

Sulaiman, Malik, OB

Supian, L. S., OB

Svede, Aiga, 3A

Tcarenkova, Elena, 20

Teixeira, António L., OQ, 1M, 32

Teixeira, Marcelo C. M., IW

Tepichín Rodríguez, Eduardo, 31, 38, 39

Texeira, D., OD

Torres, Cesar, OW, 28

Torres, $\mathrm{H} ., \mathrm{O} 3$

Tuna, Ana Rita, IP

Urban, Alexander S., 2E

Úsuga-Restrepo, J., 34, 37

Vaca Pereira G., M., OR

Vagner da Silva, Erlon, 1Q

Valencia, J. L., 10

Vallés, Juan A., OS

Vasilevskiy, Mikhail, OV

Vázquez Dorrío, Benito, 10, $2 \mathrm{U}$

Velosa, F., 05

Vieira, M., 09, OA, OD, OH

Vieira, M. A., 09, OA, OH

Vieira, P., OA

Vilardy, Juan M., OW, 29, 36

Vilarinho, Daniel, 2G

Vilhena, Henrique, 1Y, 30 
Villa, J., $1 \mathrm{~T}$

Villatoro, Joel, OR

Vujicic, Zoran, 32

Vygranenko, $Y ., O D$

Wang, Jue, 07, 2Q, 2Y

Wasige, Edward, 07, 2Q, 2Y

Watson, Scott, $2 Q$

Wawrzyńczyk, Dominika, OK

Webb, David J., 33

Williamson, Sandra, 2A

Wiśniewski, Krzysztof, OF

Wold, J. P., 19

Yebra, Ana, 13, 1V, 1X, 2M

Yebra-Pimentel, Eva, 00, 0T

Zam, Azhar, 11, 1S

Żelechower, Michał, OF

Zhang, Weikang, $2 Q$

Zubia, Joseba, OR

Zuñiga-Bedoya, J., 37

Proc. of SPIE Vol. 10453 1045301-13

Downloaded From: https://www.spiedigitallibrary.org/conference-proceedings-of-spie on 26 Apr 2023 Terms of Use: https://www.spiedigitallibrary.org/terms-of-use 


\title{
Conference Committees
}

\author{
Conference Chair \\ Manuel F. M. Costa, Universidade do Minho (Portugal) \\ International Scientific Committee
}

Amparo Pons Martí, Universidad de València (Spain)

Ana Consortini, Università degli Studi di Firenzi (Italy)

Anand Krishna Asundi, Optics and Photonics Society of Singapore

(Singapore) and Nanyang Technological University (Singapore)

Andrea Cusano, Università degli Studi di Sannio (Italy)

Andrew Moore, Herriot-Watt University (United Kingdom)

Andrés Márquez Ruiz, Universidad de Alicante (Spain)

Angel Augier Calderin, InSTEC-Instituto Superior de Tecnologías y Ciencas Aplicadas (Cuba)

Angel I. Negueruela, Universidad de Zaragoza (Spain)

Angela M. Guzman, CREOL, The College of Optics and Photonics, University of Central Florida (United States)

Asticio Vargas, Universidad de La Frontera (Chile)

Carlos Ferreira, Universidad de València (Spain)

Carlos Saavedra Rubilar, Universidad de Concepción (Chile)

Cesar Augusto Costa Vera, Escuela Politécnica Nacional (Ecuador)

Clementina Timus, National Institute for Laser, Plasma, and Radiation

Physics (Romania)

Cristiano M. B. Cordeiro, Universidade Estadual de Campinas (Brazil)

Daniel Malacara Hernández, Centro de Investigaciones en Óptica

(Mexico)

Efraín Solarte Rodriguez, RCO-Red Colombiana de Óptica (Colombia)

Eric Rosas, Centro de Investigaciones en Óptica, A.C. (Mexico)

Eugene Arthurs, SPIE

Gonçalo Figueira, Universidade Nova de Lisboa (Portugal)

Guillermo Baldwin, Pontificia Universidad Católica del Perú (Peru)

Hai-Ning Cui, Jilin University (China)

Hector Rabal, ClOp-Centro de Investigaciones Ópticas (Argentina)

Humberto Michinel, Universidad de Vigo (Spain)

Hypolito Kalinowski, Universidade Tecnológica Federal do Paraná (Brazil)

James Wyant, College of Optical Sciences, The University of Arizona (United States)

Jana B. Nieder, International Iberian Nanotechnology Laboratory (Portugal)

Jesús Lancis, Universidad Jaume I (Spain)

Joaquín Campos Acosta, CSIC-Instituto de Óptica (Spain)

João Lemos Pinto, i3N-Institute of Nanostructures, Nanomodelling and Nanofabrication (Portugal) 
João Manuel Tavares, Universidade do Porto (Portugal)

John Canning, University of Sydney (Australia)

José António Rodrigues, Universidade do Algarve (Portugal)

Jose Benito Vazquez-Dorrio, Universidad de Vigo (Spain)

José Figueiredo, Universidade do Algarve (Portugal)

José Luis Paz, CTOV (Venezuela)

José Manuel de Nunes Vicente Rebordão, Universidade Nova de Lisboa

(Portugal)

José Ramiro Fernandes, Universidade de Trás-os-Montes e Alto Douro

(Portugal)

José R. Salcedo, ATLA Lasers (Norway)

José Silva Gomes, Universidade do Porto (Portugal)

Juan G. Darias Gonzalez, Centro de Aplicaciones Tecnologicas y

Desarrollo Nuclear (Cuba)

Luciano Alberto Angel-Toro, RCO-Red Colombiana de Óptica

(Colombia)

Luis Miguel Bernardo, Universidade do Porto (Portugal)

Luis Roso, Centro de Láseres Pulsados Ultracortos Ultraintensos (Spain)

Luís Silvino Alves Marques, Universidade do Minho (Portugal)

Katrina Svanberg, University of Lund (Sweden)

Kiyofumi Matsuda, National Institute of Advanced Industrial Science and Technology (Japan)

Kim Chew Ng, Monash University (Australia)

Manuel Lopez-Amo, Universidad Pública de Navarra (Spain)

Manuel Melgosa Latorre, Universidad de Granada (Spain)

Marcelo Trivi, Universidad Nacional de Mar del Plata (Argentina)

Maria Josefa Yzuel, Universidad Autònoma de Barcelona (Spain)

Maria Luisa Calvo, Instituto de Credito Oficial (Spain)

Maria Sagrario Millan, Universidad Politècnica de Catalunya (Spain)

Mário Vaz, INEGI (Portugal)

Maité Flores-Arias, Universidad de Santiago de Compostela (Spain)

Marta Maria Duarte Ramos, Universidade do Minho (Portugal)

Miguel Gonzalez Herraez, Universidad de Alcalá (Spain)

Mikiya Muramatsu, Universidade de São Paulo (Brazil)

Mikhail I. Vasilevski, Universidade do Minho (Portugal)

Mourad Zghal, STO-Société Tunisienne d'Optique (Tunisia)

Mustafa Erol, Bozok University (Turkey)

Pablo Artal, Universidad de Murcia (Spain)

Paulo Torrao Fiadeiro, Universidade da Beira Interior (Portugal)

Paulo J. da Silva Tavares, Universidade do Porto (Portugal)

Pedro Andrés Riao, Universidad de València (Spain)

Radu Chisleag, Technical University of Bucharest (Romania)

Ramón Rodríguez-Vera, Centro de Investigaciones en Óptica, A.C. (Mexico)

Raul Rangel, Centro de Investigación Científica y de Educación Superior de Ensenada B.C. (Mexico)

Rastogi Pramod, École Polytechnique Fédérale de Lausanne

(Switzerland)

Robert Lieberman, Lumoptix, LLC (United States) 
Roger Ferlet, Université de Paris (France)

Salvador Bará, Universidad de Santiago de Compostela (Spain)

Tomas Catunda, Universidade de São Paulo (Brazil)

Sabry Abdel-Mottaleb, Ain-Shams University (Egypt)

Santiago Vallmitjana Rico, SEDOPTICA-Sociedad Española de Óptica

(Spain) and Universidad de Barcelona (Spain)

Sun Tong, City University (United Kingdom)

Toyohiko Yatagai, Utsunomiya University (Japan)

Waclaw Urbanczyk, Wroclaw University of Technology (Poland)

Zuqing Zhu, University of Science and Technology of China (China)

Program Review Committee

António Baptista, Universidade do Minho (Portugal)

António Lobo, Universidade Fernando Pessoa (Portugal)

Benito Vázquez-Dorrío, Universidad de Vigo (Spain)

Gonçalo Figueira, Universidade de Lisboa (Portugal)

João M. P. Coelho, Universidade de Lisboa (Portugal)

José António Rodrigues, Universidade do Algarve (Portugal)

Manuel F. M. Costa, Universidade do Minho (Portugal)

Manuel Melgosa Latorre, Universidad de Granada (Spain)

Mikhail I. Vasilevskiy, Universidade do Minho (Portugal)

Orlando Frazão, Universidade do Porto (Portugal)

Paulo Torrão Fiadeiro, Universidade da Beira Interior (Portugal)

Paulo J. da Silva Tavares, Universidade do Porto (Portugal)

Rogério Nunes Nogueira, Universidade de Aveiro (Portugal)

\section{Program Committee}

Alexandre Cabral, Universidade de Lisboa (Portugal)

Alicia Fernandéz Oliveras, Universidad de Granada (Spain)

Amit Garg, Acharya Narendra Dev College (India)

Ana Maria Rocha, Instituto de Telecomunicações (Portugal)

Anand Krishna Asundi, Optics and Photonics Society of Singapore

(Singapore) and Nanyang Technological University (Singapore)

Angel Augier Calderin, José Antonio Echevarría Higher Polytechnic Institute (Cuba)

Angela M. Guzman, CREOL, The College of Optics and Photonics, University of Central Florida (United States)

António Baptista, Universidade do Minho (Portugal)

António Lobo, Universidade Fernando Pessoa (Portugal)

Carla Carmelo Rosa, Universidade do Porto (Portugal)

Carlos Saavedra Rubilar, Universidad de Concepción (Chile)

Cesar Augusto Costa Vera, Escuela Politécnica Nacional (Ecuador)

Clementina Timus, National Institute for Laser, Plasma and Radiation

Physics (Romania)

Efraín Solarte Rodriguez, RCO-Red Colombiana de Óptica (Colombia)

Eric Rosas, Centro de Investigaciones en Óptica, A.C. (Mexico)

Gonçalo Figueira, Universidade de Lisboa (Portugal) 
Hai-Ning Cui, Jilin University (China)

Humberto Michinel, Universidad de Vigo (Spain)

Ireneu Dias, INESC Porto (Portugal)

Jana B. Nieder, International Iberian Nanotechnology Laboratory

(Portugal)

Joaquim Carneiro, Universidade do Porto (Portugal)

João M. P. Coelho, Universidade de Lisboa (Portugal)

João Manuel Tavares, Universidade do Porto (Portugal)

Jose Benito Vazquez-Dorrio, Universidad de Vigo (Spain)

José Figueiredo, Universidade do Algarve (Portugal)

José Luis Paz, National Polytechnic School (Venezuela)

José Luís Santos, Universidade do Porto (Portugal)

José Manuel Baptista, INESC Porto (Portugal)

José R. Salcedo, ATLA Lasers (Norway)

José Silva Gomes, Universidade do Minho (Portugal)

Lúcia Bilro, Instituto de Telecomunicações (Portugal)

Luis Miguel Bernardo, Universidade do Porto (Portugal)

Kim Chew Ng, Monash University (Australia)

Manuel F. M. Costa, Universidade do Minho (Portugal)

Manuel Joaquim Marques, Universidade do Porto (Portugal)

Mário Lima, Universidade de Aveiro (Portugal)

Naoya Wada, National Institute of Information and Communications

Technology (Japan)

Nélia Alberto, Instituto de Telecomunicações (Portugal)

Orlando Frazão, Universidade do Porto (Portugal)

Robert Lieberman, Lumoptix, LLC (United States)

Rogério Nunes Nogueira, Universidade de Aveiro (Portugal)

Susana Silva, INESC-TEC (Portugal)

Technical Chairs

António Baptista, Universidade do Minho (Portugal)

Gonçalo Figueira, Universidade de Lisboa (Portugal)

José António Rodrigues, Universidade do Algarve (Portugal)

José Figueiredo, Universidade do Algarve (Portugal)

Orlando Frazão, Universidade do Porto (Portugal)

Paulo Torrao Fiadeiro, Universidade da Beira Interior (Portugal)

Rogério Nunes Nogueira, Universidade de Aveiro (Portugal) 


\section{Introduction}

Celebrating Optics and Photonics and its outstanding positive impact in the world and in our everyday life (largely resulting from the current remarkable success and fast sustainable development in optics and photonics research, the Portuguese Society for Optics and Photonics (Portugal), SPOF - Sociedade Portuguesa para a Investigação e Desenvolvimento em Óptica e Fotónica (Portugal)) successfully organised its third triennial international conference on Applications of Optics and Photonics, 8 - 12 May, at the Universidade do Algarve in the lovely city of Faro, Portugal.

The conference was organized to foster the establishment of the widest range of cooperation projects and relationships with colleagues and institutions from all around the world, while increasing the external visibility of Portugal's optics and photonics research.

The success of the conference came from the enthusiastic commitment of the Portuguese optics and photonics community and of our friends from all over the world, and the endorsement and active support of the most important international scientific optics societies:

ICO, SPIE, EOS, RIAO; several national societies, committees, and boards: SEDOPTICA-Sociedad Española de Óptica (Spain), AMO (Mexico), STO (Tunisia), OPSS (Singapore), CVO (Venezuela), RCO (Colombia), SOFE (Ecuador), and SPF (Portugal):

other photonics and optics industries and companies, as well as the following projects and initiatives: Laserlab-Europe, iBROW, INNOVA, Laser World of Photonics, OPA, OQEJ-Springer, adLASER, MTBrandão, Lda.;

and the contribution from the authors and presenters of the 191 effectively presented talks in all domains of optics and photonics.

Of the over two hundred works presented, the conference included 32 invited lectures from world leading scientists and 7 plenary lectures, which gave an excellent overview of the state of the art in optics and photonics research across the world pointing out perspectives of future developments.

Well over one third of all 149 effective participants of the conference were students. This percentage increased to nearly 50\% among the Portuguese participants, illustrating the vitality and growth potential of research in optics and photonics in Portugal, and all over the world in general. The support of the International Commission for Optics helped the participation of seventeen students. EOS and SPIE awarded prizes, respectively, to the best student poster presentations and best student research works presented at the conference. 
At the closing ceremony of the conference the 2014 and 2015 SPOF's Best PhD Thesis in Portugal in Optic and Photonics were awarded to Hugo Martins ("Distributed and Remote Fiber Sensing Assisted by Raman Effect") and to Marta Ferreira ("Fiber Sensing Based on New Structures and Post-Processing Enhancement"), both former students of the Universidade do Porto (Portugal).

Reflecting the development of the scientific and technological research in optics and photonics in Portugal over last decades and the success of the previous editions of SPOF's conference; it was decided to change the periodicity of our AOP conferences to biannual. Therefore our next conference will be held $3-7$ June, 2019, in Lisbon, Portugal.

We are looking forward to another exciting and most enjoyable conference!

Manuel F. M. Costa 
Proc. of SPIE Vol. 10453 1045301-20 Downloaded From: https://www.spiedigitallibrary.org/conference-proceedings-of-spie on 26 Apr 2023
Terms of Use: https://www.spiedigitallibrary.org/terms-of-use 